\title{
Identification and comparison of circular RNAs in preeclampsia
}

\author{
Zepeng Ping ${ }^{\text {Equal first author, } 1}$, Ling Ai ${ }^{\text {Equal first author, } 1}$, Huaxiang Shen ${ }^{1}$, Xing Zhang ${ }^{2}$, Huling Jiang ${ }^{\text {Corresp., } 1}$, Ye Song ${ }^{\text {Corresp. } 3}$ \\ 1 Department of Obstetrics, Maternity and Child Health Care Affiliated Hospital, Jiaxing University, Jiaxing, China \\ 2 School of Biology \& Basic Medical Science, Soochow University, Suzhou, China \\ 3 Department of Obstetrics, Suzhou Municipal Hospital, Suzhou, Jiangsu, China \\ Corresponding Authors: Huling Jiang, Ye Song \\ Email address: jianghuling@zju.edu.cn, 202061224050@njtech.edu.cn
}

Background . Preeclampsia (PE) is a pregnancy-specific syndrome, belongs to the gestational hypertension diseases category and is considered among the causes of maternal and perinatal mortality and morbidity. However, the pathogenesis of PE is still vague.

Methods. In the present study, the circular RNA (circRNA) expression patterns of normal pregnant women and PE patients were investigated using whole RNA sequencing.

Results. 151 differential expressed circRNAs were identified including 121 upregulated and 30 downregulated ones. Functional and pathway enrichment analysis was conducted on the differentially expressed circRNAs using Gene Ontology and KEGG databases. The results of this analysis indicated that several crucial biological processes and pathways were enriched in PE patients. circRNA-microRNA (miRNA) interaction analysis indicated that the reported differentially expresse circRNAs may be associated with some regulatory functions through miRNAs in PE patients. Two ceRNAs networks were constructed according to the targeting relationship between circRNAs/miRNAs and miRNAs/mRNAs. One sub-network contained one upregulated circRNA, four downregulated miRNAs and five upregulated mRNAs, and another sub-network contained 10 downregulated circRNAs, 21 upregulated miRNAs and 15 downregulated mRNAs.

Conclusion . CircRNA expression patterns have been investigated and this analysis revealed their potential regulatory mechanisms in PE patients. We constructed the ceRNAs (competing endogenous RNA) to reveal the potential molecular roles of dysregulated circRNAs in the PE patients using RNA sequencing data. circRNA_13301 was the only one upregulated circRNA in ceRNA being targeted by four miRNAs. 


\section{Identification and comparison of circular RNAs in}

2 Preeclampsia

3

4

Zepeng Ping ${ }^{1, \#}$, Ling $\mathrm{Ai}^{1, \#}$, Huaxiang Shen ${ }^{1}$, Xing Zhang ${ }^{2}$, Huling Jiang ${ }^{1, *}$, Ye Song ${ }^{3, *}$

${ }^{1}$ Department of Obstetrics, Maternity and Child Health Care Affiliated Hospital, Jiaxing University, Jiaxing, China

${ }^{2}$ School of Biology \& Basic Medical Science, Soochow University, Suzhou, China ${ }^{3}$ Department of Obstetrics, Suzhou Municipal Hospital, Suzhou, Jiangsu, China Corresponding Author:

Huling Jiang ${ }^{1}$ 2468 Central South Road, Jiaxing, Zhejiang, 314000, China

Email address: jianghuling@zju.edu.cn

Ye Song ${ }^{3}$

26 Daoqian Street, Gusu District, Suzhou, Jiangsu, 215000, China

Email address: 202061224050@njtech.edu.cn

\section{Abstract}

Background. Preeclampsia (PE) is a pregnancy-specific syndrome, belongs to the gestational hypertension diseases category and is considered among the causes of maternal and perinatal mortality and morbidity. However, the pathogenesis of PE is still vague.

Methods. In the present study, the circular RNA (circRNA) expression patterns of normal pregnant women and PE patients were investigated using whole RNA sequencing.

Results. 151 differential expressed circRNAs were identified including 121 upregulated and 30 downregulated ones. Functional and pathway enrichment analysis was conducted on the differentially expressed circRNAs using Gene Ontology and KEGG databases. The results of this analysis indicated that several crucial biological processes and pathways were enriched in PE patients. circRNA-microRNA (miRNA) interaction analysis indicated that the reported differentially expresse circRNAs may be associated with some regulatory functions through miRNAs in PE patients. Two ceRNAs networks were constructed according to the targeting relationship between circRNAs/miRNAs and miRNAs/mRNAs. One sub-network contained one upregulated circRNA, four downregulated miRNAs and five upregulated mRNAs, and another sub-network contained 10 downregulated circRNAs, 21 upregulated miRNAs and 15 downregulated mRNAs. 
34 Conclusion. CircRNA expression patterns have been investigated and this analysis revealed their

35

36

37

38

39

40

41

42

43

44

45

46

47

48

49

50

51

52

53

54

55

56

57

58

59

60

61

62

63

64

potential regulatory mechanisms in PE patients. We constructed the ceRNAs (competing endogenous RNA) to reveal the potential molecular roles of dysregulated circRNAs in the PE patients using RNA sequencing data. circRNA_13301 was the only one upregulated circRNA in ceRNA being targeted by four miRNAs.

\section{Introduction}

Preeclampsia (PE) is a pregnancy-specific syndrome, belongs to the common gestational hypertension disease category, and is considered among the causes of mortality and morbidity of pregnant women (Cote et al., 2008; Jia and Li, 2019). A pregnant women is diagnosed with PE when the both of following clinical features are identified of systolic / diastolic blood pressure $\geqslant 140 / \geqslant 90 \mathrm{mmHg}$ and substantial proteinuria $\geqslant 300 \mathrm{mg}$ during 24 hours at or after 20 weeks of gestation (Milne et al., 2005; Wisner, 2019). Women with severe PE might present multisystem disturbance syndrome (Maynard et al., 2005; Abalos et al., 2013).The exact etiologyand pathogenesis of PE still remains unclear.

Numerous recent studies have illustrated that a variety of non-coding RNAs (ncRNAs) are related to the pathogenesis of pregnancy diseases (Huang et al., 2018; Jiao et al., 2018; Wang et al., 2018; Cheng et al., 2019; Yang et al., 2019), wherein, circular RNAs (circRNAs) belong to one type of ncRNAs with various of biological features, such as evolutionary conservation, structural stability and tissue specificity so on (Jeck et al., 2013;Memczak et al., 2013), which play crucial roles in biological functions of certain diseases (Jia and Li, 2019). The biological roles of circRNAs were gradually exposed from studies on various cancers and many other diseases, and the prevalent hypothesis for their roles include the following: miRNA sponges, RNA-binding proteins (RBPs), gene transcription regulation and mRNA translation mediators (Kristensen et al., 2019). Some dysregulated circRNAs were associated with the development and the progression of numerous diseases (Bai et al., 2019;Li et al., 2019a;Li et al., 2019b;Zhou et al., 2019), while other dysregulated circRNAs have presented the potential to be used as biomarkers (Shao et al., 2019; Tian et al., 2019;Wang et al., 2019;Wu et al., 2019). Regarding the role of circRNAs in PE several studies have been reported from other groups, who identified crucial roles of specific circRNAs in the pathogenesis of PE by regulating ceRNA. Furthermore, some circRNAs have been suggested as potential novel biomarkers for PE. Most of these 
65

66

67

68

69

70

71

72

73

74

75

76

77

78

79

80

81

82

83

84

85

86

87

88

89

90

91

92

93

94

circRNAs were identified from the trophoblast cell or human placenta using human circRNA microarray or using publicly available datasets from the Gene Expression Omnibus (GEO) databaseand meta-analyzing them with bioinformatics techniques (Zhou et al., 2018; Liu et al., 2019). In addition, Hsa_circ_0007121 was reported to mediate the progression of PE via miR182-5p/placental growth factor (PGF) axis (Gai et al., 2020). CircLRRK1 was identified to ssuppress the proliferation, migration and invasion of trophoblast cells via miR-2233p/PI3K/AKT axis (Tang et al., 2020). circ_0001438 aggravated the dysfunctions of human villous trophoblasts by mediating the miR-942/NLRP3 axis (Li et al., 2020). circCRAMP1L (Zhang et al., 2020), CircSFXN1 (Zhang et al., 2020), Circ_0085296 (Zhu et al., 2020) were reported to play important roles in the pathogenesis of PE.

In the present study, the expression profiles of circRNAs in PE and healthy pregnant women were investigated with whole transcriptome sequencing and the regulatory mechanism of circRNAs in PE was explored. The findings of this study will contribute to the understanding of the etiology and the pathogenesis of PE, especiallythe regulatory mechanism of circRNAs in PE.

\section{Materials \& Methods}

\section{Ethics statement}

This study was approved by the Ethics Committee of the Faculty of Medicine from the Maternity and Child Health Care Affiliated Hospital, Jiaxing University (Ethical Application Ref: 201947). Informed consent was signed in agreement with subjects in the study.

\section{Sample collection}

A total of 40 pregnant women (20 cases with PE patients and 20 normal pregnant women) from the Maternity and Child Health Care Affiliated Hospital, Jiaxing University were recruited for this study (Table 1). All pregnant women were strictly screened according to the clinical features of PE cases to exclude other causes (Wisner, 2019). The whole blood samples (3.0ml) were collected from the PE patients and normal pregnant women, and leukocyte cells were isolated from the collected whole blood using the Red Blood Cell Lysis Buffer kit (Tiangen, Beijing,

3 China). The cells were transferred into Eppendorf tubes with TRIzol reagent (Invitrogen

94 Carlsbad, CA, USA). Total RNA was quantified with a NanoDrop ND 2000 spectrophotometer 
95 96

97

98

99

100

101

102

103

104

105

106

107

108

109

110

111

112

113

114

115

116

117

118

119

120

121

122

123

124

125

and RNA integrity was assessed using an Agilent Bioanalyzer 2100(Agilent Technologies Santa Clara, CA, USA).

\section{rRNA-depleted RNA sequencing}

First, Total RNAs was extracted from three PE cases and three normal controls using the TRIzol reagent. After quality control, the TruSeq Stranded Total RNA with Ribo-Zero Gold kit (for plants, use the TruSeq Stranded Total RNA LT-(with Ribo-Zero Plant) kit ) was used to digest ribosomal RNA and added a breaking reagent to break the RNA into short fragments. Later the disrupted RNA was used as a template to synthesize one-strand cDNA with six-base random primers and then prepare a two-strand synthesis reaction system to synthesize two-strand cDNAwith dUTP instead of dTTP. Followed with the UNG enzymatic method to digest a strand containing dUTP, and only the first cDNA strand with different linkers in the linking strand was retained. The first cDNA strand was purified and repaired. A-tailed was connected to the sequencing adapter, and then fragment size was selected for finally PCR amplification. RNA Integrity Number threshold of 7 was applied to construct the libraries using TruSeq Stranded Total RNA Library Prep Kit (Illumina, USA). Six libraries were sequenced on the HiSeqTM4000 sequencing platform. Sequencing analysis was conducted by Shanghai OE Biotech, Shanghai, China. All raw data has been uploaded to Sequence Read Archieve: PRJNA665923.

\section{CircRNA prediction, expression analysis and interactions research}

After performing quality control tests on the raw fastq files, the extracted clean reads were aligned to the human reference genome (GRCh38.p12) using hisat2 tool. The CIRI software (2015) was used to scan for paired chiastic clipping signals (CIRI: an efficient and unbiased algorithm for de novo circular RNA identification). circRNA sequences were predicted based on the junction reads and GT-AG cleavage signals. RPM algorithm was applied to calculate the relative expression of circRNAs normalizing the matched read number with the transcripts' length. Miranda software was used to predict the targeting interactions between miRNAs and circRNAs.

\section{Differential screening analysis and Functional Analysis}


126

127

128

129

130

131

132

133

134

135

136

137

138

139

140

141

142

143

144

145

146

147

148

149

150

151

152

153

154

155

156

DESeq tool (http://www.bioconductor.org/packages/release/bioc/html/DESeq.html) was applied to perform differential expression analysis and to calculate the p-values and fold-change of each identified transcript. The differentially expressed (DE) circRNAs were screened with standard of $\mathrm{p}$ value threshold $<0.05$ and fold change threshold $>2$. Enrichment analysis of the DE circRNAs against the Gene Ontology (GO) and Kyoto Encyclopedia of Genes and Genomes (KEGG) was conducted with using the Hypergeometric Distribution Test method. DIANA-mirPath was utilized to predict miRNA targets.

\section{ceRNA network analysis}

Based on the ceRNA (competing endogenous RNA) regulatory mechanisms (Salmena et al., 2011), the miRNA targets (miRNA-mRNA or miRNA-circRNA targets) were predicted, and then circRNA-mRNA regulation pairs were predicted according with the guilty by association method (Zhang et al., 2017; Zhang et al.,2018). At the same time, the correlation of regulation relation to expression value was calculated. The ceRNA networks of upregulated circRNAdownregulated miRNA-upregulated mRNA and downregulated circRNA-upregualted miRNAdownregulated mRNA were constructed with Cytoscape software version 3.7.1.

\section{Real-time PCR assay}

7 DE circRNAs were randomly selected for validation with real-time PCR. The divergent primers were designed according to flanking sequences of junction sites of the circRNAs (Table 2). RNA was extracted for 20 PE samples and 20 normal control samples. cDNAs was produced as the template by cDNA Synthesis SuperMix (Transgen Biotech, Beijing, China). Real-time PCR was performed on Real-Time PCR Detection System (Bio-Rad,USA) with Universal SYBR Green Supermix (Bio-Rad, USA) according to the procedure. $\beta$-actin was used as internal standard control and delta delta CT method $\left(2^{-\Delta \Delta \mathrm{Ct}}\right)$ method was applied to calculate the expression level of circRNAs. All experiments were repeated in three times.

\section{Results}

\section{Characterization of circRNAs from the $P E$ and normal pregnant women}

After the circRNA prediction with the CIRI software, the identified circRNAs were compared with human circRNAs reported in CircBase (http://www.circbase.org/) with 14674 of them being 
157 identified in both PE and healthy pregnant women. 6842 circRNAs had been recorded in

158 CircBase data with 7832 circRNAs being newly introduced ones (Figure 1A). The scaled relative 159 expressions of circRNAs in the six samples of the present study were displayed with a heatmap

160 (Figure 1B). The p-values and fold changes reported from the differential expression analysis of 161 circRNAs were visualized in the form of a volcano plot (Figure 1C). After a stringent screening 162 strategy, adjusting $\mathrm{p}$-values for multiple testing (adjusted $\mathrm{p}$ value $<0.05$; fold change, $>2$ or $<$

163 0.5), 120 significantly upregulated and 31 significantly downregulated circRNAs were identified 164 in the leukocytes of PE women compared with control groups (Figure 1D). These findings 165 indicated that the relative expression of several circRNAs was altered in the leukocytes of the 166 pregnants with PE, indicating that these DE circRNAs may be associated with the etiology and 167 pathogenesis of PE.

168

169 GO annotation of DE circRNAs

170 To gain further insights into the biological processes that were potentially mediated by these DE 171 circRNAs in PE, GO annotation analysis was used to determine the functions of differentially 172 expressed circRNAs using the parental transcripts. $64 \mathrm{GO}$ terms were significantly enriched 173 (FDR < 0.05) in the source transcripts of the DE circRNAs. The top 30 biological processes 174 related GO terms that were found to be enriched in upregulated circRNAs included terms such as 175 negative regulation of cytokine-mediated signaling pathway, positive regulation of macrophage 176 tolerance induction, negative regulation of macrophage cytokine production, response to 177 peptidoglycan, regulation of protein complex disassembly, negative regulation of toll-like 178 receptor signaling pathway, negative regulation of protein complex disassembly, negative 179 regulation of interleukin-6 production, response to exogenous dsRNA and negative regulation of 180 innate immune response. The top 30 molecular function related $\mathrm{GO}$ terms that were found to be enriched in upregulated circRNAs included: protein heterodimerization activity, SH3 domain 182 binding, receptor binding, cadherin binding, RNA binding, magnesium ion binding, protein 183 tyrosine phosphatase activity, protein heterodimerization activity, ubiquitin protein ligase 184 binding and protein kinase binding (Figure 2A). The top 30 biological processes related GO 185 terms that were found to be significantly enriched in downregulated circRNAs included: 186 regulation of immune response, cell surface receptor signaling pathway, immune response, 187 adaptive immune response, neutrophil degranulation and transcription. The top 30 molecular 
188 function related GO terms that were found to be significantly enriched in downregulated 189 circRNAs included: protein homodimerization activity, DNA binding and metal ion binding 190 (Figure 2B). From the GO enrichment analysis of the source transcripts of DE circRNAs, many 191 important GO terms were revealed including immune response and protein homodimerization 192 activity.

193

\section{KEGG enrichment of DE circRNAs}

195 The source transcripts of DE circRNAs were further used to identify significantly enriched 196 KEGG pathways in the list of DE circRNAs. 55 KEGG pathways (FDR < 0.05) were revealed to 197 be significantly enriched in the DE circRNAs. The top 20 enriched pathways enriched in upregulated circRNAs included: glycerolipid metabolism, glycerophospholipid metabolism, 199 mRNA surveillance pathway, RNA degradation, RNA transport, Influenza A, neurotrophin

200

201

202

203

204

205

206

207

208

209

210

211

212

213

\section{4}

215

216

217

218 signaling pathway and endocytosis (Figure 3). The top 20 enriched pathways in downregulated circRNAs included osteoclast differentiation and human cytomegalovirus infection (Figure 3). These results revealed that some DE circRNAs were enriched in lipid metabolism and virus infection.

\section{Validation of circRNA expression}

A set of DE circRNAs(hsa_circ_0001289, hsa_circ_0008311, hsa_circ_0004960, hsa_circ_0003753, hsa_circ_0001861, hsa_circ_0006719 and hsa_circ_0005806) was selected to validate the findings of the differential expression analysis using real-time PCR. Similar trends in the expression profile of all these circRNAs were identified between real-time PCR and highthroughput sequencing data (Figure 4A-G). Moreover, the junction sites of these circRNAs were further validated with reverse transcription PCR and Sanger sequencing (Figure 4H-N). These results confirmed the validity of the sequencing data from the PE patients.

\section{CircRNA-miRNA interaction analysis}

The expression level of mRNAs is regulated by circRNAs through miRNA mediation. Therefore, a comprehensively analysis of the interactions between circRNA and miRNA will help to explore the potential regulatory mechanisms of circRNAs in PE. A circRNA-miRNA network was constructed based on the differentially expressed circRNAs and differentially expressed 
219 miRNAs identified from the PE and visualized with Cytoscape (Figure 5A). The results showed 220 that each circRNA could sponge several miRNAs and each miRNA can have several target 221 circRNAs. KEGG pathway enrichment analysis was conducted on the mRNA which are 222 predicted as miRNA targets to provide further insights into the biological processes of mRNAs 223 mediated by the differential expressed circRNA through miRNAs. The results indicated that the 224 miRNA target transcripts were enriched in signaling pathway of calcium, insulin secretion, 225 prolactin, biotin metabolism and GnRH (Figure 5B).

\section{circRNA-miRNA-mRNA network re-construction}

228

Two ceRNAs networks were re-constructed according to the targeting/interaction relationship between circRNAs/miRNAs and miRNAs/mRNAs to investigate the potential regulatory mechanism of DE circRNAs in the etiology and pathogenesis of PE. The p-values threshold for the of correlation coefficient of the relative expressions was set to 0.05 , and the threshold for absolute value of Pearson correlation coefficient was set to 0.7. One subnetwork included one upregulated circRNA, four downregulated miRNAs and five upregulated mRNAs (Figure 6A), and another subnetwork included 10 downregulated circRNAs, 21 upregulated miRNAs and 15 downregulated mRNAs (Figure 6B, C). These interacted circRNA-miRNA-mRNA networks could contribute to further exploring the regulatory mechanism of DE circRNAs in the pathogenesis of PE.

\section{Discussion}

Recent studies have accumulated evidence that circRNAs, a novel class of non-coding RNAs, are associated with several crucial biological roles, such as miRNA sponges, RNA-binding protein and translation (Kristensen et al., 2019). Among roles, the one of miRNA sponges was comprehensively identified in the progression and the pathogenesis of diverse of cancers. In these cases circRNAs were found to act as ceRNAs via adsorbing miRNAs to regulate the expression levels of their target genes. In addition, numerous circRNAs have been suggested as novel biomarkers for early diagnosis of diseases (Tian et al., 2019; Wu et al., 2019). However, to the best of our knowledge, there exist limited reports about the role of circRNAs in the etiology and pathogenesis of PE. In present study, the DE patterns of circRNAs were explored in blood samples of PE patients compared to normal pregnant women with 121 circRNAs found to be 
250 upregulated and 30 downregulated in PE patients. These statistically significant differentially 251 expressed circRNAs in PE patients indicated that circRNAs might play an important regulatory 252 role in the pathogenesis of PE. Furthermore, using the parental transcripts of these circRNAs to 253 explore the potential molecular functions and signaling pathways, we identified that the DE 254 circRNAs were significantly enriched to various biological processes and signaling pathways. $255 \mathrm{PE}$ is a serious and common pregnancy complication, which is considered one of the causes for 256 increased morbidity and mortality in pregnancies (Antwi et al., 2013). It is known that many 257 biological processes (immune maladaptation, inadequate placental development, trophoblast 258 invasion, placental ischemia, oxidative stress and thrombosis) have been associated with the 259 etiology and the pathogenesis of PE (Zhou et al., 2018), but the mechanism underlying PE development was still unclear. circRNAs is a novel type short non-coding RNAs with circular structure and they have been reported to play crucial roles in the progression and pathogenesis of 262 diverse diseases (Zhang et al., 2017). The ceRNA hypothesis was comprehensively applied to explore the molecular pathogenesis of various diseases (Salmena et al., 2011) including PE. 180 DE circRNAs were identified from the placentae of severe preeclampsia, and many of them were associated to the vasodilation and the regulation of blood vessel size (Deng et al., 2019). $49 \mathrm{DE}$ circRNAs were identified in the placental tissues of PE, and this group of DE circRNAs was enriched in in MAPK signaling pathway (Ou et al., 2019). Another 49 DE circRNAs were identified from the placental tissue of PE women compared with healthy pregnant women with these playing important roles in cellular regulation via sponging miRNAs (Zhou et al., 2018). Circulating circRNAs may also have some predictive/diagnostic value for PE (Zhang et al., 2016). Most of these studies were carried out in the placental tissue of PE and the expression

272 levels of some circRNAs found to be significantly changing in the development of PE patients.

273 Much fewer studies involve analysis of peripheral blood samples from PE patients.

274 In the present study, we constructed the ceRNAs to reveal the potential molecular roles of 275 dysregulated circRNAs in the PE patients using RNA sequencing data from the PE patients vs. 276 healthy pregnant women. circRNA_13301 was the only one upregulated circRNA in ceRNA 277 network, which contained five upregulated mRNAs (centrosomal protein 295, epithelial 278 membrane protein 1, butyrophilin like 3, matrix metallopeptidase 19, LIM and calponin 279 homology domains 1) being targeted by four miRNAs (hsa-miR-3200-5p, hsa-miR-4660, hsa280 miR-5001-5p and hsa-miR-3166). Another ceRNA network contained 10 downregulated 
281

282

283

284

285

286

287

288

289

290

291

292

293

294

295

296

297

298

299

300

301

302

303

304

305

306

307

308

309

310

311

312

313

314

315

316

317

circRNAs, 21 upregulated miRNAs and 15 downregulated mRNAs. KEGG pathway enrichment analysis found that these downregulated mRNAs were enriched into nicotine addiction, cholinergic synapse, cell adhesion molecules and calcium signaling pathway. These novel mRNAs mediated by differential expressed circRNAs by sponging miRNAs, which roles played in the pathogenesis will be validated in the further research.

Our study is the systematic profiling of ceRNAs in leukocyte of PE patients and revealed the global ceRNA network integration in PE. These data will provide novel clues to understand the etiology and pathogenesis of PE from the dysregulated circRNAs.

\section{Conclusions}

In summary, our findings revealed the the circRNA expression patterns in PE patients and their possible regulatory mechanisms. This study provides useful information for exploring the potential roles of circRNAs in the etiology and pathogenesis of PE.

\section{Acknowledgements}

We thank Lin He's Academician Workstation of New Medicine and Clinical Translation for its guidance and help. The authors would like to thank Dr. Qidong Zu (OE Biotech, Inc., Shanghai, China, http://www.oebiotech.com/) for assistance with the bioinformatics analysis of Wholetranscriptome sequencing.

\section{References}

Abalos E, Cuesta C, Grosso AL, Chou D, Say L. 2013. Global and regional estimates of preeclampsia and eclampsia: a systematic review. European Journal of Obstetrics \& Gynecology And Reproductive Biology 170, 1-7.

Antwi E, Groenwold R, Janssen K, Browne J, Klipstein-Grobusch, K, Agyepong I, Koram K, Franx A, Grobbee D. 2013. Predictors of pregnancy induced hypertension in an urban low resource setting. Pregnancy Hypertens 3, 97.

Bai N, Peng E, Xia F, Wang D, Li X, Li X. 2019. CircABCC2 Regulates Hepatocellular Cancer Progression by Decoying MiR-665. J Cancer 10, 3893-3898.

Cheng D, Jiang S, Chen J, Li J, Ao LF, Zhang Y. 2019.Upregulated long noncoding RNA Linc00261 in pre-eclampsia and its effect on trophoblast invasion and migration via regulating miR-558/TIMP4 signaling pathway.Journal of Cellular Biochemistry 120, 13243-13253.

Cote AM, Firoz T, Mattman A, Lam EM, Von Dadelszen P, Magee LA. 2008. The 24-hour urine collection: gold standard or historical practice? American Journal of Obstetrics And Gynecology 199.

Deng N, Lei D, Huang J, Yang Z, Fan C, Wang S. 2019. Circular RNA expression profiling identifies hsa_circ_0011460 as a novel molecule in severe preeclampsia. Pregnancy Hypertens 17, 216225. 
318

319

320

321

322

323

324

325

326

327

328

329

330

331

332

333

334

335

336

337

338

339

340

341

342

343

344

345

346

347

348

349

350

351

352

353

354

355

356

357

358

359

360

361

Huang Z, Du G, Huang X, Han L, Han X, Xu B, Zhang Y, Yu M, Qin Y, Xia Y, Wang X, Lu C. 2018. The enhancer RNA lnc-SLC4A1-1 epigenetically regulates unexplained recurrent pregnancy loss (URPL) by activating CXCL8 and NF-kB pathway. EBioMedicine 38, 162-170.

Jeck WR, Sorrentino JA, Wang K, Slevin MK, Burd CE, Liu JZ, Marzluff WF, Sharpless NE. 2013. Circular RNAs are abundant, conserved, and associated with ALU repeats. Rna 19, 141-157.

Jia N, Li J. 2019. Role of Circular RNAs in Preeclampsia. Dis Markers 2019, 7237495.

Jiao S, Wang SY, Huang Y. 2018. LncRNA PRNCR1 promoted the progression of eclampsia by regulating the MAPK signal pathway. Eur Rev Med PharmacolSci 22, 3635-3642.

Kristensen LS, Andersen MS, Stagsted LVW, Ebbesen KK, Hansen TB, Kjems J. 2019. The biogenesis, biology and characterization of circular RNAs. Nat Rev Genet 20, 675-691.

Li H, Jin X, Liu B, Zhang P, Chen W, Li Q. 2019a.CircRNA CBL.11 suppresses cell proliferation by sponging miR-6778-5p in colorectal cancer. BMC Cancer 19, 826.

Li R, Wu B, Xia J, Ye L, Yang X. 2019b. Circular RNA hsa_circRNA_102958 promotes tumorigenesis of colorectal cancer via miR-585/CDC25B axis. Cancer Manag Res 11, 6887-6893.

Liu SW, Xie X, Lei HJ, Zou BY, Xie L. 2019. Identification of Key circRNAs/lncRNAs/miRNAs/mRNAs and Pathways in Preeclampsia Using Bioinformatics Analysis. Medical Science Monitor 25, 1679-1693.

Li X, Yang R, Xu Y, Zhang Y, 2020. Circ_0001438 participates in the pathogenesis of preeclampsia via the circ_0001438/miR-942/NLRP3 regulatory network. Placenta, 104.

Maynard SE, Venkatesha S, Thadhani R, Karumanchi SA. 2005. Soluble Fms-like tyrosine kinase 1 and endothelial dysfunction in the pathogenesis of preeclampsia. Pediatr Res 57, 1R-7R.

Memczak S, Jens M, Elefsinioti A, Torti F, Krueger J, Rybak A, Maier L, Mackowiak SD, Gregersen LH, Munschauer M, Loewer A, Ziebold U, Landthaler M, Kocks C, Le Noble F, Rajewsky N. 2013. Circular RNAs are a large class of animal RNAs with regulatory potency. Nature 495, 333-338.

Milne F, Redman C, Walker J, Baker P, Bradley J, Cooper C, De Swiet M, Fletcher G, Jokinen M, Murphy D, Nelson-Piercy C, Osgood V, Robson S, Shennan A, Tuffnell A, Twaddle S, Waugh J. 2005. The pre-eclampsia community guideline (PRECOG): how to screen for and detect onset of pre-eclampsia in the community. British Medical Journal 330, 576-580.

Ou Y, Liu M, Zhu L, Deng K, Chen M, Chen H, Zhang J. 2019. The expression profile of circRNA and its potential regulatory targets in the placentas of severe pre-eclampsia.Taiwan J ObstetGynecol 58, 769-777.

Tang R, Zhang Z, Han W. 2020. CircLRRK1 targets miR-223-3p to inhibit the proliferation, migration and invasion of trophoblast cells by regulating the PI3K/AKT signaling pathway. Placenta, 104 (2021): 110-118.

Salmena L, Poliseno L, Tay Y, Kats L, Pandolfi PP. 2011. A ceRNA hypothesis: the Rosetta Stone of a hidden RNA language? Cell 146, 353-358.

Shao Y, Tao X, Lu R, Zhang H, Ge J, Xiao B, Ye G, Guo J. 2019. Hsa_circ_0065149 is an Indicator for Early Gastric Cancer Screening and Prognosis Prediction. PatholOncol Res Jul: 263(3).

Gai SK, Sun L, Wang HY, Yang P. 2020. Circular RNA hsa_circ_0007121 regulates proliferation, migration, invasion, and epithelial-mesenchymal transition of trophoblast cells by miR-1825p/PGF axis in preeclampsia. Open Med (Wars), 15 (1): 1061-1071.

Tian JH, Xi XH, Wang J, Yu JJ, Huang Q, Ma R, Zhang X, Li H, Wang LB. 2019.CircRNA hsa_circ_0004585 as a potential biomarker for colorectal cancer.Cancer Management And Research 11, 5413-5423.

Peer) reviewing PDF | (2020:08:51833:3:1:NEW 25 Mar 2021) 
362

363

364

365

366

367

368

369

370

371

372

373

374

375

376

377

378

379

380

381

382

383

384

385

386

387

388

389

390

391

392

393

394

395

396

397

398

399

Vlachos IS, Zagganas K, Paraskevopoulou MD, Georgakilas G, Karagkouni D, Vergoulis T, Dalamagas T, Hatzigeorgiou AG. 2015. DIANA-miRPath v3.0: deciphering microRNA function with experimental support. Nucleic Acids Res 43, W460-466.

Wang S, Zhang X, Li Z, Wang W, Li B, Huang X, Sun G, Xu J, Li Q, Xu Z, Xia Y, Wang L, Zhang Q, Li Q, Zhang L, Chen J, Wu Y, Cao J, Xu P, Zhang D, Xu H, Xu Z. 2019. Circular RNA profile identifies circOSBPL10 as an oncogenic factor and prognostic marker in gastric cancer. Oncogene 38, 6985-7001.

Wang XQ., Li Y, Su X, Zhang L, Liu CM, Liu H, Ma X, Xia H. 2018.Haplotype-based association of two SNPs in miR-323b with unexplained recurrent spontaneous abortion in a Chinese Han population. J Cell Physiol 233, 6001-6017.

Wisner K. 2019. Gestational Hypertension and Preeclampsia.MCN Am J Matern Child Nurs 44, 170.

Wu JH, Li JQ, Liu H, Yin JW, Zhang MJ, Yu ZB, Miao HJ. 2019. Circulating plasma circular RNAs as novel diagnostic biomarkers for congenital heart disease in children. Journal of Clinical Laboratory Analysis 33.

Yang HL, Zhang HZ, Meng FR, Han SY, Zhang M. 2019. Differential expression of microRNA-411 and $376 \mathrm{c}$ is associated with hypertension in pregnancy. Brazilian Journal of Medical And Biological Research 52.

Zhang X, Zhu M, Yang R, Zhao W, Hu X, Gan J. 2017. Identification and comparison of novel circular RNAs with associated co-expression and competing endogenous RNA networks in pulmonary tuberculosis. Oncotarget 8, 113571-113582.

Zhang YG, Yang HL, Zhang YP, Shi JZ, Chen RG, Xiao XQ. 2020. CircSFXN1 regulates the behaviour of trophoblasts and likely mediates preeclampsia. Placenta, 101: 115-123.

Zhang YG, Yang HL, Long Y, Li WL. 2016. Circular RNA in blood corpuscles combined with plasma protein factor for early prediction of pre-eclampsia. BJOG 123, 2113-2118.

Zhang Y, Yang H, Zhang Y, Shi J, Chen R. 2020. circCRAMP1L is a novel biomarker of preeclampsia risk and may play a role in preeclampsia pathogenesis via regulation of the MSP/RON axis in trophoblasts. BMC Pregnancy Childbirth, Oct 27;201(1).

Zhou W, Wang H, Wu X, Long W, Zheng F, Kong J, Yu B. 2018. The profile analysis of circular RNAs in human placenta of preeclampsia. ExpBiol Med (Maywood) 243, 1109-1117.

Zhou W, Wang H, Yang J, Long W, Zhang B, Liu J, Yu B. 2019. Down-regulated circPAPPA suppresses the proliferation and invasion of trophoblast cells via the miR-384/STAT3 pathway. Biosci Rep 39.

Zhu HL, Niu X, Li QH, Zhao YH, Chen X, Sun HS, 2020. Circ_0085296 suppresses trophoblast cell proliferation, invasion, and migration via modulating miR-144/E-cadherin axis. Placenta, 97, 1825.

Zhang X, Zhu M, Hu X. 2018. Integrated miRNA and mRNA expression profiling to identify mRNA targets of dysregulated miRNAs in pulmonary tuberculosis. Epigenomics 2018 08;108(8). 


\section{Table 1 (on next page)}

The information of patients with PE and normal control groups. 
1 Table 1 The information of patients with PE and normal control groups.

\begin{tabular}{llll}
\hline Parameter & PE $(\mathrm{n}=20)$ & Healthy controls $(\mathrm{n}=20)$ & P value \\
\hline Age (years) & $27.59 \pm 4.20$ & $27.65 \pm 2.45$ & $\mathrm{~ns}$ \\
Gestational weeks & $36.23 \pm 2.81$ & $36.88 \pm 2.71$ & $\mathrm{~ns}$ \\
Systolic blood pressure $(\mathrm{mmHg})$ & $168.91 \pm 13.25$ & $116.55 \pm 13.18$ & $* * *$ \\
Diastolic blood pressure (mmHg) & $109.91 \pm 10.94$ & $69.8 \pm 9.89$ & $* * *$ \\
Urine protein $(\mathrm{g} / 24 \mathrm{~h})$ & $2.73 \pm 1.69$ & $0.09 \pm 0.06$ & $* * *$ \\
\hline
\end{tabular}

2 The two groups were matched by age and gestational week. ns, $\mathrm{P}>0.05$ and ***, $\mathrm{P}<0.01$. 
Table 2 (on next page)

Primers for validation of circRNAs. 
1 Table 2 Primers for validation of circRNAs.

\begin{tabular}{lll}
\hline Primername & Sequence (5'-3') & Length (bp) \\
\hline hsa_circ_0001289-F & AACAGAGTCAGCATCAGAGC & 20 \\
hsa_circ_0001289-R & AGTGGCATCTATTATTGAA & 19 \\
hsa_circ_0008311-F & GTTTCTGGTTCTCAGGAT & 18 \\
hsa_circ_0008311-R & TCAATGAGAGGTCCCATCTGG & 21 \\
hsa_circ_0004960-F & TGAAAGCAAGTCACTAGAGAT & 20 \\
hsa_circ_0004960-R & CCAAAGAGGGAGCCAGATGT & 20 \\
hsa_circ_0003753-F & CTTGAAGTTTTACTACTGAG & 20 \\
hsa_circ_0003753-R & CCACGGTGACATTGGCTG & 18 \\
hsa_circ_0001861-F & AATCAAGAAGCGTGGGATCC & 20 \\
hsa_circ_0001861-R & TGCTCCACCTCACAGTTC & 18 \\
hsa_circ_0006719-F & TTACACCCAGTGCCTCTGAC & 20 \\
hsa_circ_0006719-R & TGTCCGCAGGCAACCCTT & 18 \\
hsa_circ_0005806-F & CGGCCACCTTTGAGGCTCT & 19 \\
hsa_circ_0005806-R & GAGAACAAGGAGGGGTGGTG & 20 \\
\hline
\end{tabular}

2 


\section{Figure 1}

Identification of differentially expressed circRNAs from PE patients.

(A) Total circRNAs and known circRNAs compared with circBase. (B) Heatmap showing the differentially expressed circRNAs from the PE patients (P1, P2 and P3) compared with normal healthy individuals ( $\mathrm{C} 1, \mathrm{C} 2$ and $\mathrm{C} 3$ ). (C) Volcano plot showing the differentially expressed circRNAs from the six samples. (D) Statistical results of differentially expressed circRNAs in PE patients compared with healthy individuals. 
A

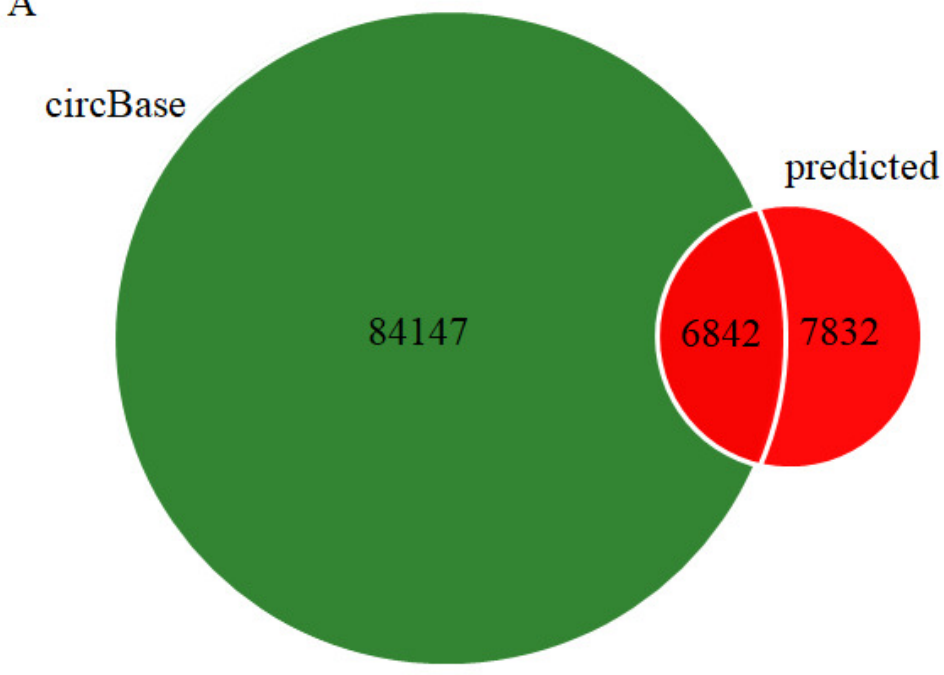

B

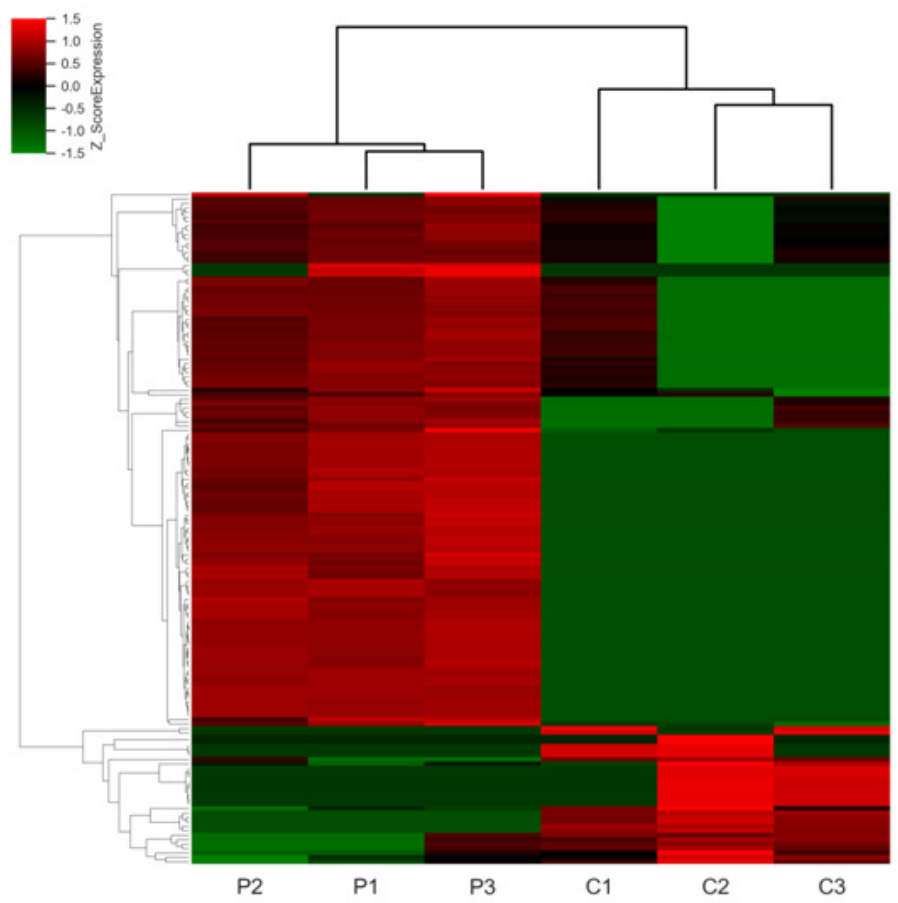

C

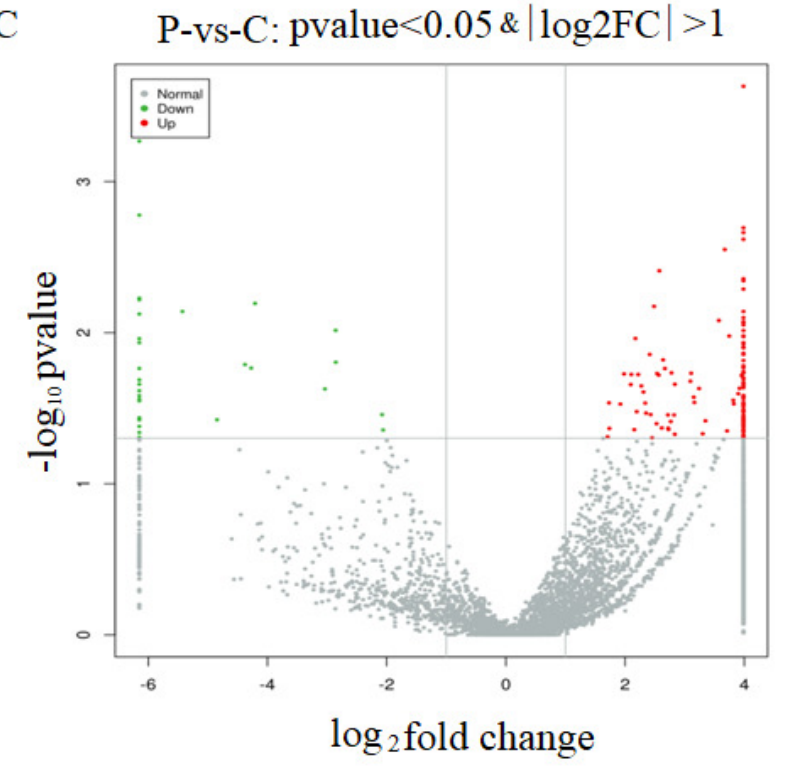

D

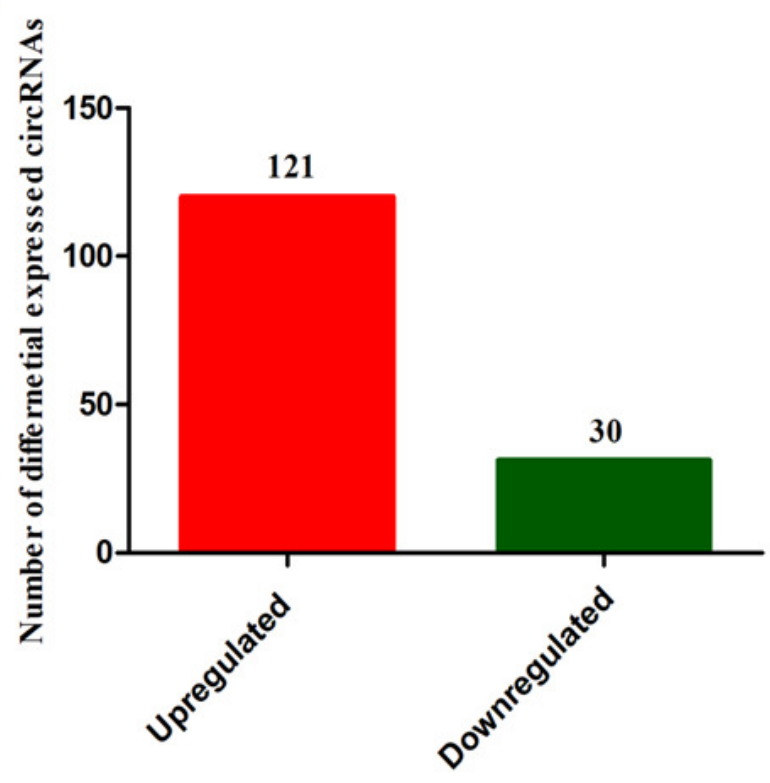


Figure 2

GO enrichment analysis of differentially expressed circRNAs using their parental transcripts.

(A) GO enrichment analysis of upregulated circRNAs. (B) GO enrichment analysis of downregulated circRNAs. 
A

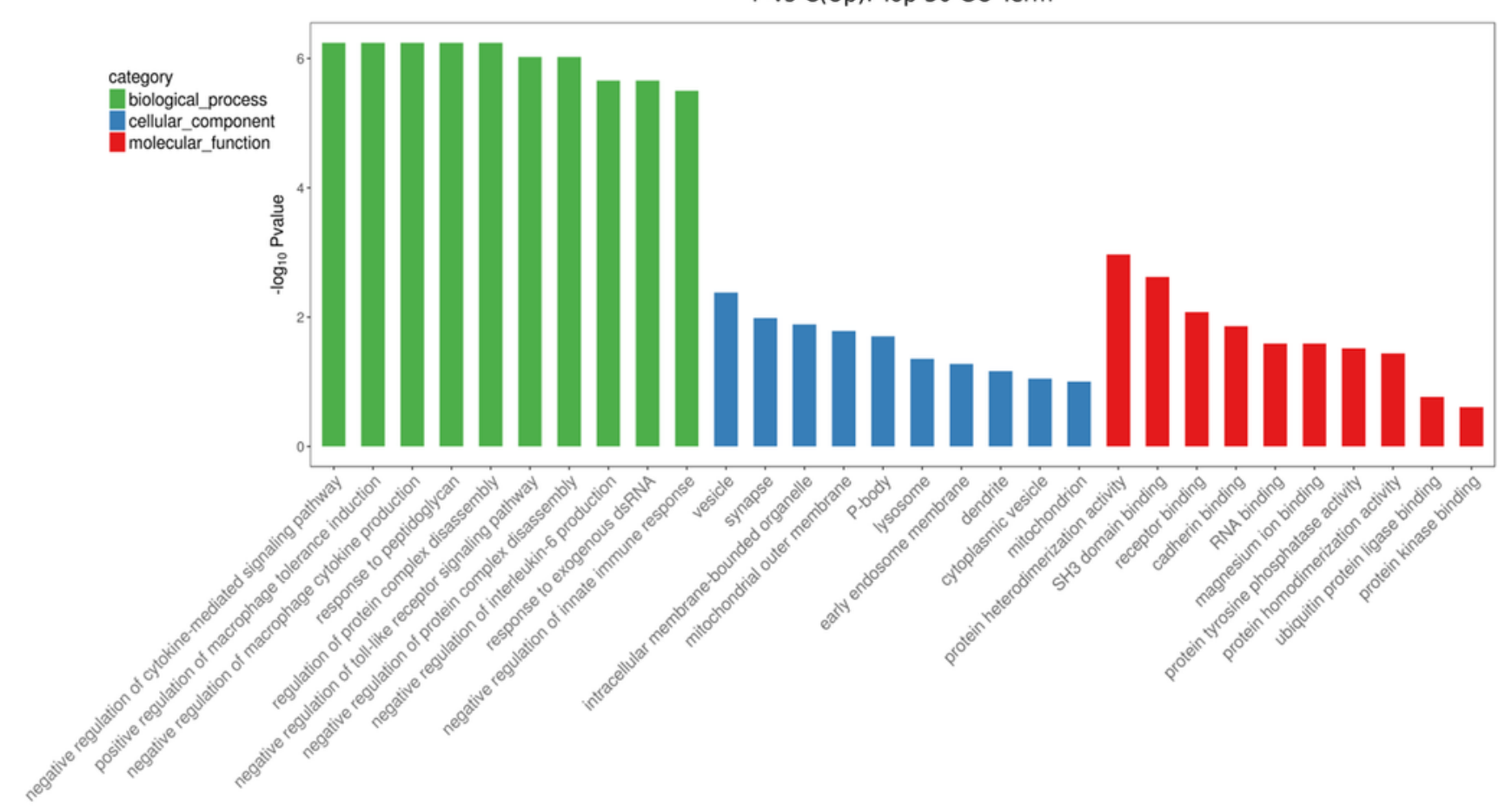

B

P-vs-C(Down): Top 30 GO Term

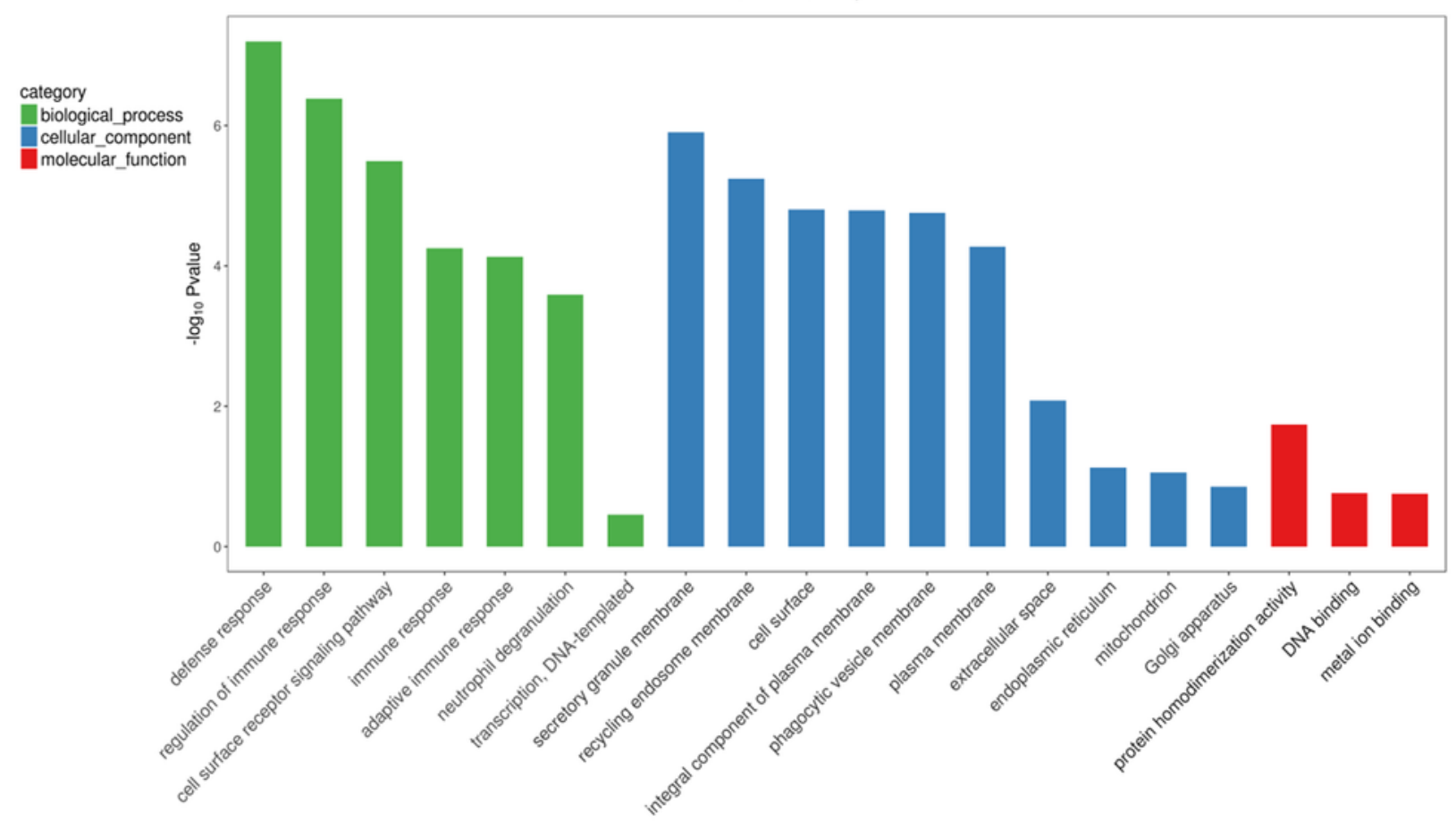


Figure 3

\section{KEGG enrichment analysis of differentially expressed circRNAs using their parental transcripts.}

KEGG enrichment analysis of differential expressed circRNAs using their parental transcripts.

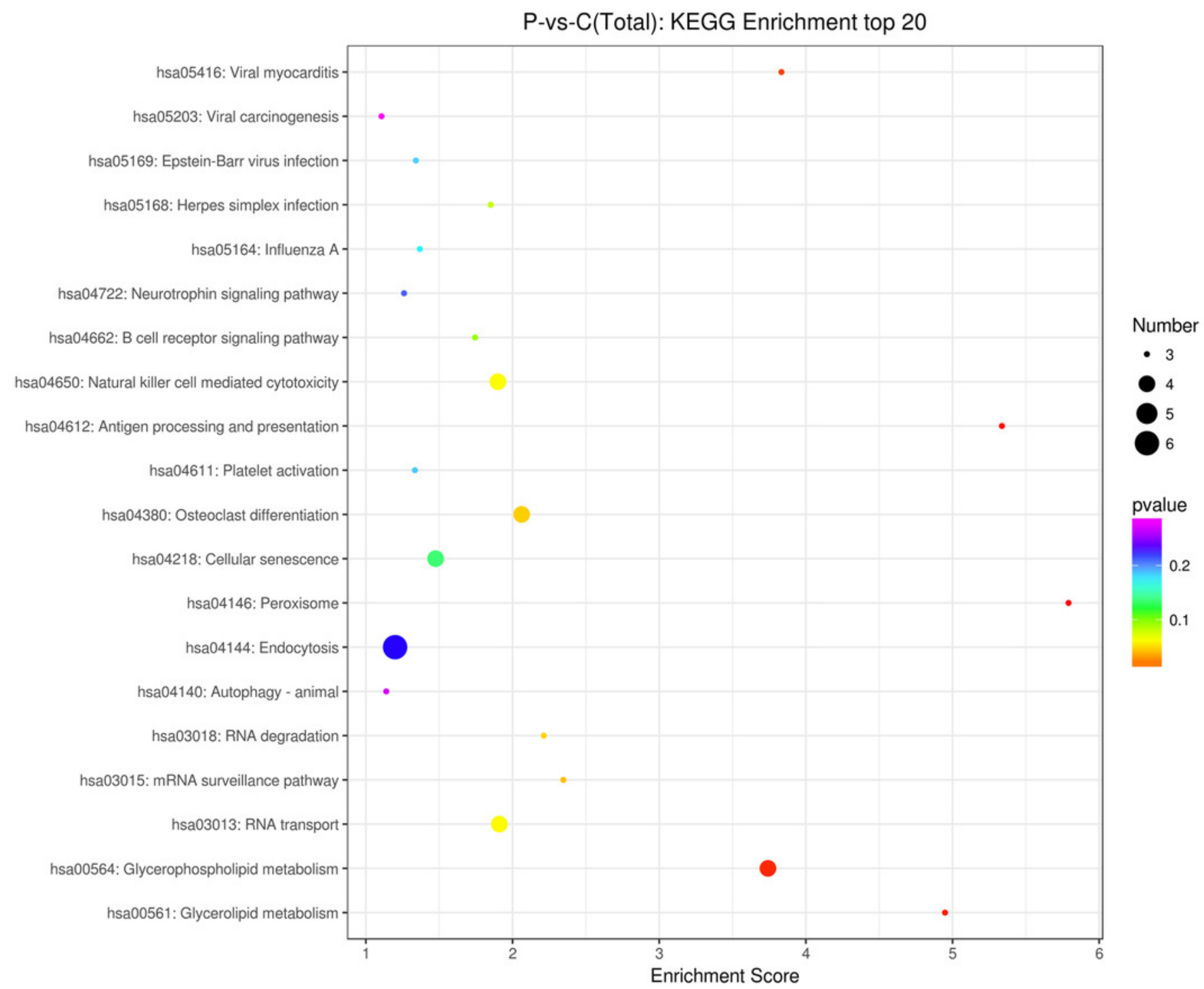


Figure 4

circRNAs validation with real-time PCR and reverse transcription PCR.

(A-G) Validation of differentially expressed circRNAs (hsa_circ_0001289, hsa_circ_0008311, hsa_circ_0004960, hsa_circ_0003753, hsa_circ_0001861, hsa_circ_0006719 and hsa_circ_0005806) from the 20 PE patients and healthy individuals with real-time PCR. (H-N) Validation of junction site of the circRNAs with reverse transcription PCR. 

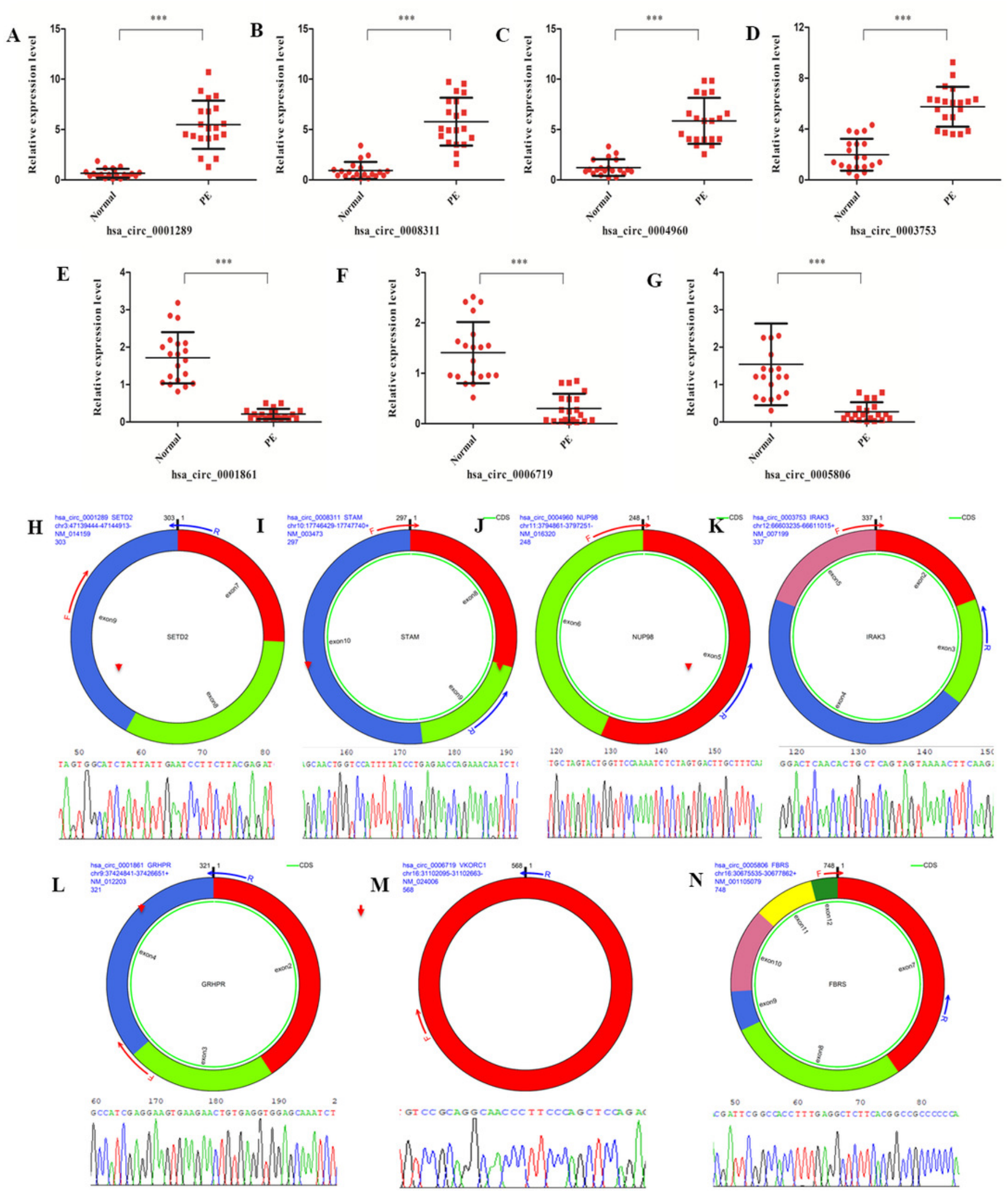
Figure 5

The co-expression network of circRNAs and miRNAs and the associated signaling pathways analysis with the targets of co-expressed miRNAs.

(A) The network of differentially expressed circRNAs with miRNAs. (B) The enriched signaling pathways were predicted with the using the targets of co-expressed miRNAs.
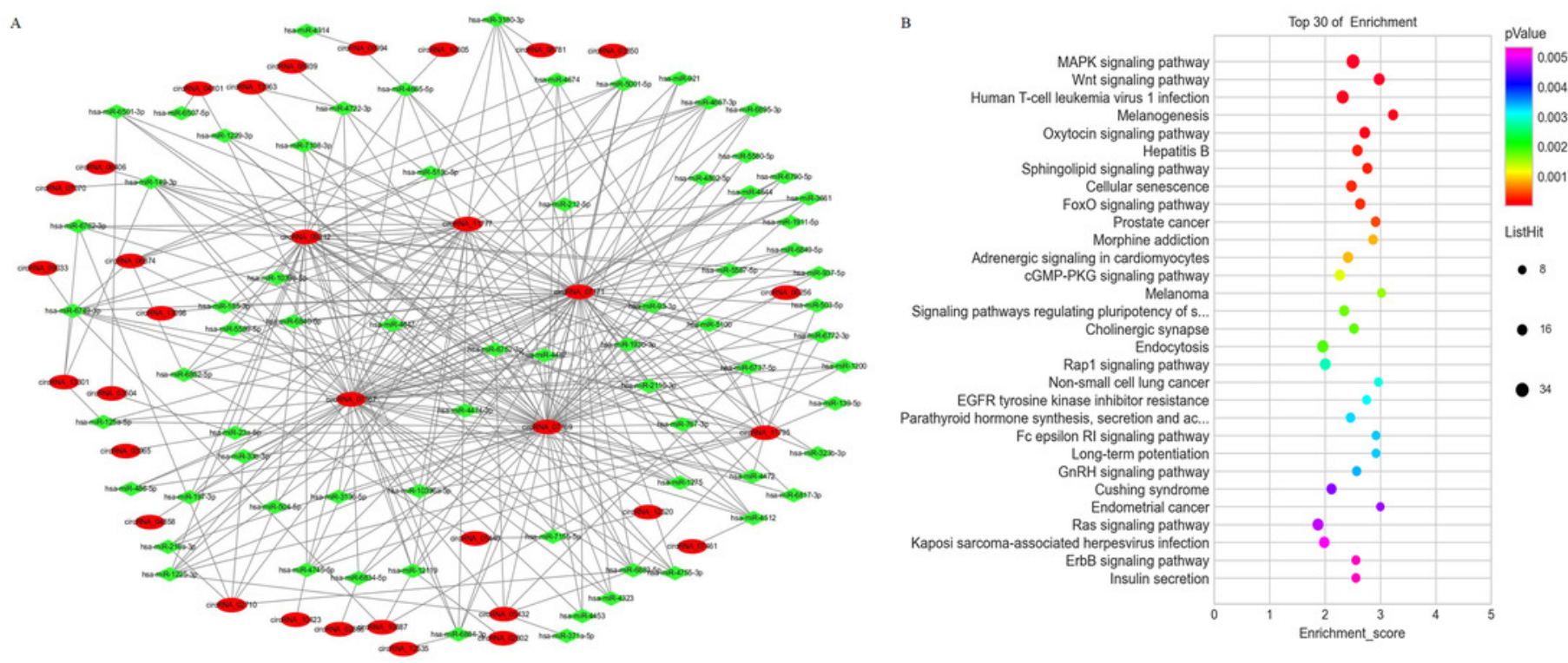
Figure 6

Re-construction of ceRNA networks with differentially expressed circRNAs, miRNAs and mRNAs.

(A) The ceRNA network including upregulated circRNAs, downregulated miRNAs and upregulated mRNAs. (B.C) The ceRNA network including downregulated circRNAs, upregulated miRNAs and downregulated mRNAs.

A

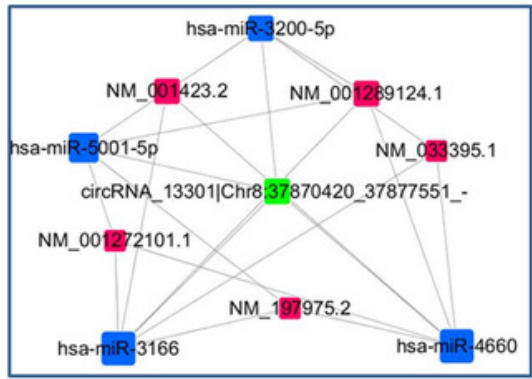

B

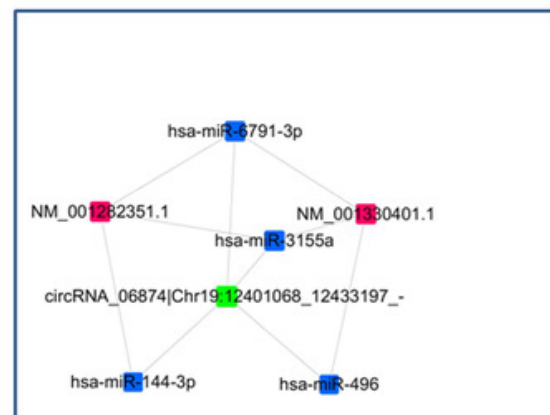

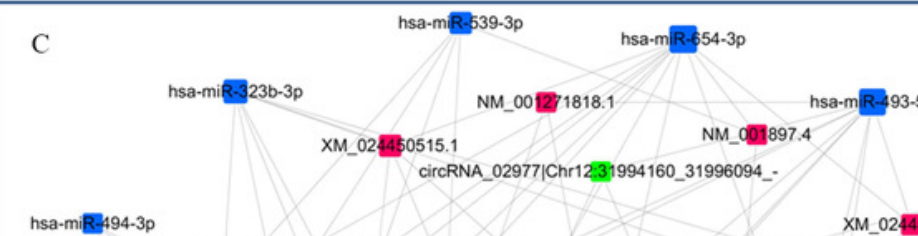

hsa-miR-494-3p circRNA_13098|Chr7:142056830_142082571 +

XM_024452218. NM_0012D2473.1

circRNA_11777|Chr629890246_29944616_+ XM_017009252.1

circRNA_07169|Chr19:54218645_54276003_-

XM_01 1537926.3
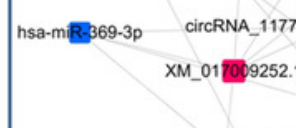

circRNA_09212|Chr22:42516011_42577098

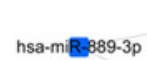
XM_02 $44 \$ 1951.1$

CircRNA_14233|Chr9: 114354636_114355285_circRNA_07171|Chr19:54222943_54280299_ NM_00 1256573.1 XM_01 5 9116.2 nsa-miR-411-3p circRNA_11795|Chr631271999_31356957_. hsa-m R-654-5p hsa-miR-410-3p NM_Q15532.4 XM_017003468.1 NM_00 1185011.1 hsa-mR-379-5p 